\title{
The Dynamic Response of Dense 3 Dimensionally Printed Polylactic Acid
}

\author{
H. O. Agu' ${ }^{1}$ - A. Hameed ${ }^{1}$. G. J. Appleby-Thomas ${ }^{1}$ - D. C. Wood ${ }^{1}$
}

Received: 28 January 2019 / Accepted: 14 May 2019 / Published online: 22 May 2019

(c) The Author(s) 2019

\begin{abstract}
Polylactic acid (PLA) is commonly used as a feedstock material for commercial 3D printing. As components manufactured from such material become more commonplace, it is inevitable that some of the resultant systems will be exposed to high strain-rate/impact events during their design-life (for example, components being dropped or even involved in a high-speed crash). To this end, understanding the shock properties of polylactic acid, in its role as a major raw material for 3D printed components, is of particular importance. In this work, printed samples of PLA were deformed by one-dimensional shock waves generated via the plate impact technique, allowing determination of both the Hugoniot Equation of State (EOS) and shear strength of the material. Both linear and non-linear EOS forms were considered in the $\mathrm{U}_{\mathrm{S}}$-Up plane, with the best-fit found to take the general form $U_{S}=1.28+3.06-1.09 U_{p}^{2}$ in the $U_{s}-U_{p}$ plane, consistent with other polymers. Use of lateral Manganin gauges embedded in the material flow allowed consideration of lateral stress evolution at impact pressures ranging from 0.3 to $4.0 \mathrm{GPa}$. Shear strength was observed to increase with impact stress, however, with minimal strengthening behind the shock front. Deviation of the measured stress from the predicted elastic measurement (corresponding to the PLA's Hugoniot Elastic Limit) was observed at longitudinal stress of $0.90 \pm 0.05 \mathrm{GPa}$, within range of polymeric materials of similar characteristics - the first time this important parameter has been measured for PLA. As a result, this material characterisation will allow numerical modellers to accurately predict the structural response of PLA-based components/structures against high strain rates such as impacts or drops.
\end{abstract}

Keywords Polylactic acid $\cdot$ Hugoniot elastic limit $\cdot$ Equations of state $\cdot$ Polymer

\section{Introduction}

Polylactic acid is one of the major raw materials used in a filament deposition modelling (FDM) desktop 3D printer. The application of PLA has metamorphosed from the production of simple prototypes to manufacturing of finished/ end-use products such as medical implants in the form of screws, plates and anchors [1], subject to a range of loading conditions. It is therefore important to understand the shock response of printed PLA. More importantly, understanding of the material's hydrodynamic and constitutive equations of state will enable the development of mathematical models for numerical simulations [2]. Although there are a number of studies on the response of printed PLA, [3-5] within the

\section{H. O. Agu}

henry.agu@ cranfield.ac.uk

1 Centre for Defence Engineering, Cranfield University, Shrivenham SN6 8LA, UK quasi-static loading regime, there is- to the author's knowledge- - no existing study focused on the dynamic response of PLA under high strain rate conditions. However, usefully/ as a potential point of comparison, a number of studies have already been conducted on the shock response of similar polymeric materials such as polyethylene (PE) [6], Polymethylmethacrylate (PMMA) [7], polyvinylchloride (PVC) [6] and polytetrafluoroethylene (PTFE) [6].

One of the earlier investigation conducted by Carter and Marsh [8] at the Los Alamos National Laboratory investigated the shock behaviour of over 20 polymers, showing certain similarities in the dynamic response of such materials. It was observed that at high pressure (20-30 GPa), there is a change in the slope of the shock velocity, $\left(U_{s)}\right.$ and particle velocity, $\left(U_{p}\right)$, curve suggesting a phase transformation. This was attributed to the re-ordering of the polymer structure due to the nature of the compression at sufficiently high pressure [8]. More or less, a rearrangement of the chains at the molecular level resulting in a large volume change at high 
pressure was postulated. Another observable trend was the inability of the experimentally measured data in the $U_{s}-U_{p}$ plane to extrapolate to the zero-pressure ultrasonic sound speed measurement [8]. In contrast with metals, the bulk sound speed, $C_{B}$ is usually below the y-intercept, giving polymers a non-linear Hugoniot. For example, Millet et al. [6] investigated the shock response of 4 common semi-crystalline thermoplastic polymers: PE,PTFE, PVC and PCTFE [6] and observed that all four polymers had a linear response in the $U_{s}$ and $U_{p}$, curve in common with other polymeric materials, represented in the form described in Eq. 1. However, when interpolated from the measured bulk sound speed on the y-intercept, a non-linear equation presented in the form described in Eq. 2 was obtained.

$U_{s}=c_{0}+S u_{p}$,

$U_{s}=c_{0}+S U_{p}+R U_{p}^{2}$.

The values of the $c_{0}$ and $S$ in the Hugoniot equations are empirical constants, correlated to the bulk sound speed $c_{B}$ and the first pressure derivative of the bulk modulus respectively [9].

This trend has been reported in a number of studies and has been attributed to variation between the forces in the $\mathrm{C}-\mathrm{C}$ backbone (of higher magnitude) and the forces between the adjacent chains in the polymer altering the deformation pattern along the chains and backbone [8]. As such, in contrast with metals, the chemistry of polymer is key to understanding its dynamic response. Essentially, the carbon backbone in polymers (chain) forms a strong bond with itself compared with bonds between adjacent polymer chains [8]. Hence, as the shock propagates, smaller/weaker forces in the adjacent chain collapse even more rapidly than the forces in the $\mathrm{C}-\mathrm{C}$ backbone. Usefully, the interaction between functional groups and or atoms (steric effect) plays a significant role in polymer response as well as the geometric arrangement (Tacticity) of these groups. As an example, greater physical interaction between adjacent polymer chains is required in PE molecule due to its relatively open nature. Similarly, the addition of a single chlorine atom in PVC to the basic polyethylene monomer reduces the stress required for the atoms to slide past each other during shock loading. This is because of the sheathing effect of the large chlorine atoms (compared to hydrogen) upon the polymer chain, resulting in an over-all smoother shape that allows easier passage of the polymer chains [6]. However, the presence of chlorine (in PVC) and fluorine atoms (in PCTFE) also increases the influence of electrostatic repulsion arising from increased electronegativity. Following on from this, it is likely that the presence of an oxygen atom in the side group chain of the PLA, presented in Fig. 1, would influence the shock response of PLA. As the slightly positive sections

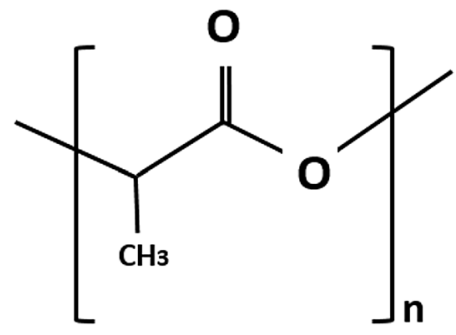

Fig. 1 Representative monomer structure of Polylactic acid

(carbon and hydrogen) are attracted to the negative sections of the chains, the stress required to break the bond increases with a consequent effect on the polymer strength.

Further, as the polymer is compressed and the chains move closer together, physical interaction between the molecules (tangling or steric effect) due to the shape of the polymer increases the stress required to move the molecules together. Though reported particularly for polymers with open chain morphology such as PE [6] with dangling side groups, the addition of an electronegative atom to the chains increase the susceptibility for entanglement. Such an interplay between electronegative repulsion and tangling determines the polymer strength under dynamic (shock) loading. While the degree at which these factors influence polymer strength is yet to be fully established, it has been suggested that repulsive forces are more dominant in the fluorinated polymer such as PTFE and PCTFE, in contrast with hydrocarbon polymers controlled majorly by tangling. Interestingly, PLA has an open chain morphology similar to $\mathrm{PE}$ and the presence of a $\mathrm{CH}_{3}$ side chain group should allow a greater degree of interaction (steric effect) between the adjacent polymer chains. In the same vein, the presence an electronegative atom (oxygen), while even not as strongly electronegative as the chlorine and fluorine atoms in PVC and PCTFE, means it is likely that the interaction of both factors could increase the strength of PLA. In this work, the dynamic shock behaviour of a 3D printed PLA was investigated using manganin stress gauges. These gauges, of thickness, ca. $25 \mu \mathrm{m}$ and embedded in the material flow, measure the longitudinal and lateral stresses induced in the samples. The data obtained were reduced to determine the Hugoniot Equations of state of the material and the shear strength — key data to allow for subsequent high strain-rate/ pressure simulations.

\section{Material Production}

The PLA filament, of diameter $2.85 \mathrm{~mm}$, used in this study was obtained commercially and supplied in an airtight bag to prevent degradation from atmospheric humidity [10]. A $50 \mathrm{~mm}$ diameter, $10 \mathrm{~mm}$ thick disc was designed on 
Computer-aided Design (CAD) software and imported as an STL file into an Ultimaker 3D printer with a $0.4 \mathrm{~mm}$ print core for printing via the Filament Deposition Modelling (FDM) desktop 3D printer (Ultimaker 3 extended). The 3D printing technique creates parts by heating filaments above its glass transition temperature before laying successive layers on the print bed based on input print parameters. Although, there are no standard parameter values for desired printing quality in the literature, the effect of certain parameters has been reported. As an example, Sood et al. [11] investigated the effect of printing parameters such as orientation, layer thickness, and air gaps on the quality of printed parts by studying their flexural, tensile, and impact strength. Schopper et al. [12] investigated the effect of build direction on the compression properties of FDM built parts and observed higher compressive modulus and yield strength of built parts in the horizontal direction in comparison to the specimens built in the vertical direction [13]. In turn, Caneiro et al. [5] investigated the effect of the layer thickness by comparing samples produced with layer thicknesses of 0.20 and $0.35 \mathrm{~mm}$ and results have shown higher tensile stress with an increasing layer thickness which was attributed to the lower number of interfaces between filaments. In the same work, the degree of infill (a printer setting which controls the density of the built parts) was reported to have a strong impact on the mechanical performance of printed samples. A difference in both modulus and tensile strength of more than $250 \%$ was reported when the infill density varied from 20 to $100 \%$ [5]. As such, for the investigations here/ to ensure an optimum print, a $100 \%$ infill density was used with a print speed of $70 \mathrm{~mm} / \mathrm{s}$ employed. The target samples were finished with an impervious top and bottom layer to prevent percolation of epoxy used in the target preparation. Other print parameters are summarised in Table 1. A total of

Table 1 Print parameters for the production of $10 \mathrm{~mm}$ thick PLA on Ultimaker FDM printer

\begin{tabular}{ll}
\hline Parameter & Value \\
\hline Layer height & $0.1 \mathrm{~mm}$ \\
Line width & $0.35 \mathrm{~mm}$ \\
Wall thickness & $0.5 \mathrm{~mm}$ \\
Z Seam alignment & Random \\
Top/bottom pattern & Concentric \\
Top/bottom thickness & $1 \mathrm{~mm}$ \\
Infill & $100 \%$ \\
Print speed & $70 \mathrm{~mm} / \mathrm{s}$ \\
infill speed & $40 \mathrm{~mm} / \mathrm{s}$ \\
Wall speed & $30 \mathrm{~mm} / \mathrm{s}$ \\
Travel speed & $250 \mathrm{~mm} / \mathrm{s}$ \\
Printing temp & $200{ }^{\circ} \mathrm{C}$ \\
Build plate temperature & $60{ }^{\circ} \mathrm{C}$ \\
\hline
\end{tabular}

eight samples were fabricated from a roll of $2.85 \mathrm{~mm}$ PLA from Spool Work. The samples were printed vertically with the layers oriented perpendicular to the shock direction. All test specimens were printed under identical conditions to maintain consistency in all samples.

In order to evaluate the consistency of the print quality, weight and diameter of each sample were obtained. The weight was found to be consistent with a tolerance of $\pm 0.2 \mathrm{~g}$ while the average density was $1.14 \pm 0.02 \mathrm{~g} / \mathrm{cc}$, slightly lower than the nominal bulk PLA density of $1.24 \mathrm{~g} / \mathrm{cc}$. This could be attributed to the presence of voids [3] which are a function of the input parameters during the printing process. The diameter was consistent with a tolerance of $\pm 0.01 \mathrm{~mm}$.

\section{Experimental Method}

One of the methods commonly used to produce a shock wave in materials is the plate-impact technique. The plateimpact technique involves accelerating a flat and parallel plate into a similar target (surfaces were polished to a tolerance of $<10 \mu \mathrm{m}$ ) [14-16] using a single stage gas gun. Simultaneous impact of all elements of the flyer and target impact surfaces leads to inertial confinement over very short (micro-second) timescales and the establishment of a one-dimensional state-of-strain within the materials. The gas gun employed for these experiments has a 50-mm bore, with a 5-m barrel and is situated at the Defence Academy of the UK, Shrivenham. A schematic of the gun set-up is presented in Fig. 2. Well characterised materials such as Copper alloy (C101) and Aluminium (1050A) flyers, whose equations of state are known were employed to impact PLA targets at impact velocities in

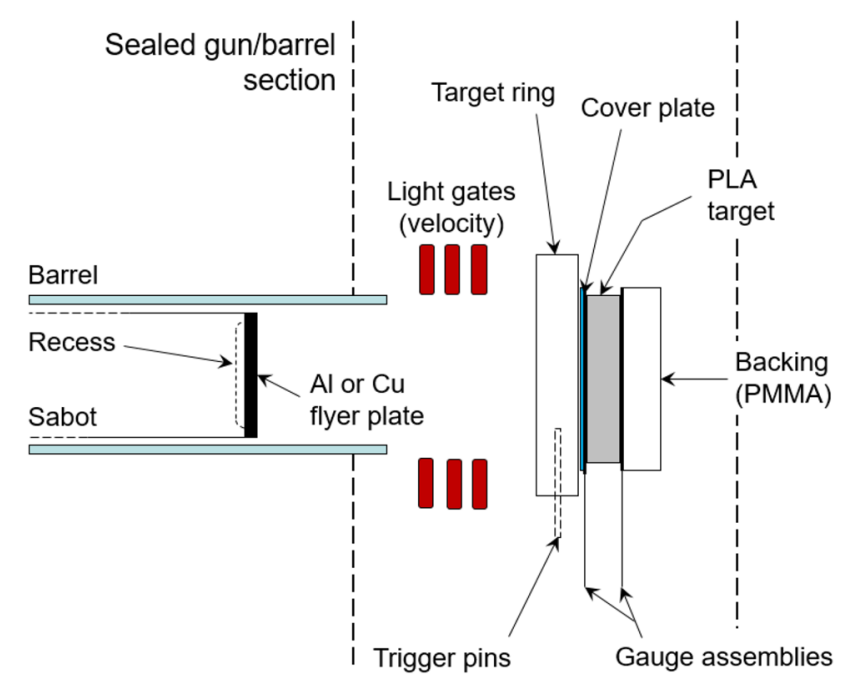

Fig. 2 Schematic representation of the mounting configuration of longitudinal target arrangement in a plate impact experiment 
the range $300-1000 \mathrm{~m} / \mathrm{s}$. The projectile impact velocity was recorded via a series of light gates at known separation immediately prior to impact. Further, target rings were employed to support the target and ensure alignment experimentally with respect to the flyer, on a sacrificial barrel extension to ensure good flyer-target alignment.

Manganin longitudinal stress gauge (Vishay MicroMeasurement of Type LM-SS-125CH-048) of $48.0 \pm 1.0 \%$ grid resistance in Ohms resistance were used to determine the stresses in the material. The gauges were placed at the front and rear of the target separated by a 50 micron Mylar (25 microns on each side) as illustrated in Fig. 2 to protect and insulate the gauges. $10 \mathrm{~mm}$ thick PMMA was used at the rear gauge to trap reflection of the shock wave. All the components were held neatly together with a slow curing Locite 0151 HYSOL epoxy-patch adhesive. $1 \mathrm{~mm}$ thick aluminium and copper cover plates (depending on the impactor material) were employed at the target surface to protect the gauge from being damaged by the impactor.

The shear strength of the material was also measured by obtaining the lateral component of the stress $\left(\sigma_{y}\right)$, from which the shear strength behind the shock front is calculated from the relationship in Eq. 3,

$2 \tau=\sigma_{x}-\sigma_{y}$.

Embedded lateral Manganin gauges (Vishay MicroMeasurement of Type J2M-SS-580SF-025) were introduced into sectioned samples, $4 \mathrm{~mm}$ from the impact surface as shown in Figs. 3 and 4. This depth was chosen to allow sufficient time for the shock to equilibrate/to resolve the elastic precursor (if any) before shock arrival. The samples were held firmly with a Locite 24-h epoxy in a special jig for a minimum of $12 \mathrm{~h}$. In some experiments, longitudinal and lateral gauges were combined at impact velocities between 200 and $900 \mathrm{~m} / \mathrm{s}$ to obtain additional $U_{s}-U_{p}$ data point on the Hugoniot. Errors were minimised by ensuring proper gauge alignment in the sectioned lateral halves of the target [17].

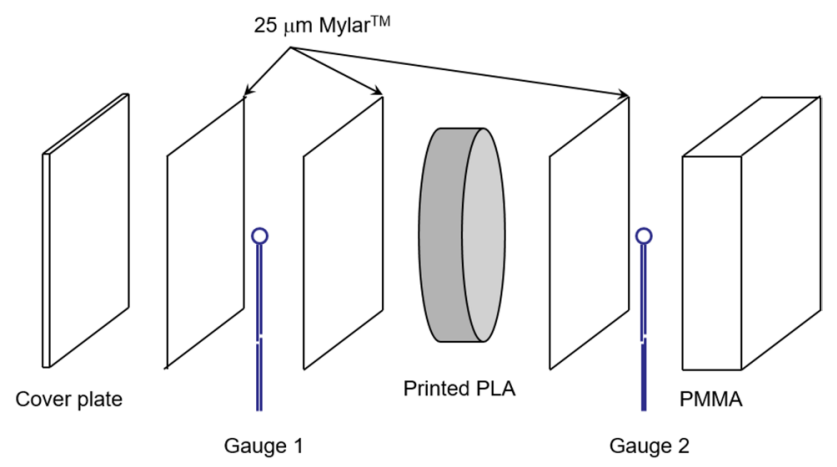

Fig. 3 Schematic representation of target configuration in plate impact experiment showing expanded view

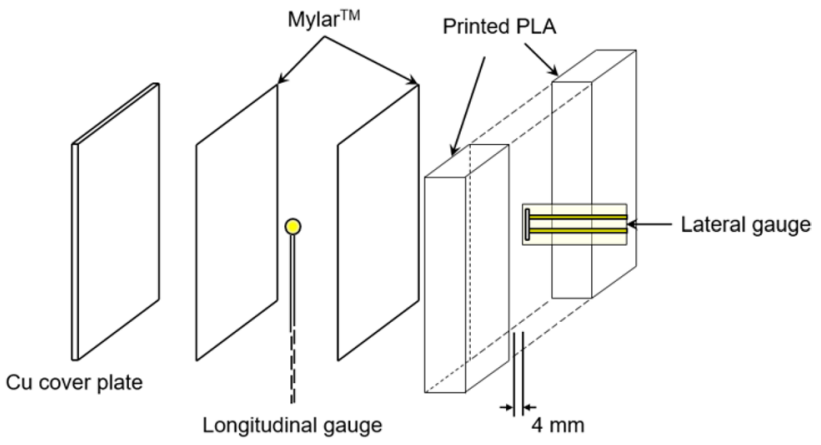

Fig. 4 Schematic representation of target configurations with longitudinal and lateral gauge positions

The densities of the printed PLA samples were measured using an XS 105 dual range excellence machine from Mettler Toledo. A density reduction relative to the initial material density $(1.24 \mathrm{~g} / \mathrm{cc})$ was observed in all samples as a result of the pores generated between layers during the printing process. In addition, shear $\left(C_{S}\right)$ and longitudinal $\left(C_{L}\right)$ sound velocities were measured ultrasonically using $1 \mathrm{MH}_{\mathrm{Z}}$ quartz transducers with a Panemetrics 5077PR pulse receiver in the pulse-echo configuration. Employing the isotropic relation for wave speed in Eq. 4 [18], the bulk sound $c_{B}$ was obtained. Key elastic material properties of the printed PLA are summarised in Table 2.

$C_{B}=\sqrt{C_{L}^{2}-\frac{4}{3} C_{S}^{2}}$

The FDM 3D printing technique has been shown to exhibit anisotropic behaviour dependant on print orientation [19]. As such, the wave speeds were measured perpendicular to the print direction and at different points on the surface to check that the resultant wave speed is consistent. The measured longitudinal sound speed for the printed PLA sample was $1860 \mathrm{~m} / \mathrm{s}$ - corresponding to a bulk sound speed of $1250 \mathrm{~m} / \mathrm{s}$ (Table 2). This appears to be below the result obtained by Parker et al. [20] who employed the pulse-echo technique to determine the bulk sound speed and the acoustic impedance of PLA to be around $2260 \mathrm{~m} / \mathrm{s}$. Variations between the measured sound speed measurements is consequently tentatively attributed to the presence of voids arising from the 3D printing manufacturing technique employede.g. FDM when compared with a cast PLA. Even though the

Table 2 Relevant elastic material property for printed PLA measured perpendicular to the printing direction

\begin{tabular}{llll}
\hline$\rho_{0} \mathrm{~g} / \mathrm{cm}^{3}$ & $C_{L}(\mathrm{~mm} / \mu \mathrm{s})$ & $C_{s}(\mathrm{~mm} / \mu \mathrm{s})$ & $C_{B}(\mathrm{~mm} / \mu \mathrm{s})$ \\
\hline 1.14 & $1.86 \pm 0.02$ & 1.19 & 1.25 \\
\hline
\end{tabular}


pores are microscopic, small void contents can influence the sound speed in a mixture as the rate at which sound travels is lowered due to the need for waves to travel around the pores [21].

\section{Results and Discussion}

A total of 6 experimental shots were undertaken at impact velocities between 200 and $1000 \mathrm{~m} / \mathrm{s}$ using 5 and $10 \mathrm{~mm}$ thick aluminium and copper flyers against $\sim 10 \mathrm{~mm}$ thick printed PLA targets. Figure 5 shows representative shock profiles of the printed PLA for 3 selected impact velocities ranging from $213-925 \mathrm{~m} / \mathrm{s}$ corresponding to impact pressure between 0.3 and 4.0 GPa. Essentially, as touched on earlier by ensuring that all impact faces (target and projectile) are parallel and finished to a tolerance of $<10 \mu \mathrm{m}$, planar shock waves are rapidly established in both the target and impactor due to the material being inertially confined on impact. The resultant shocks are $1 \mathrm{D}$ in nature and are maintained until release arrival from free surfaces (interfaces). Key features can be interpreted from the shock profile obtained from the experiments. An initial pressure rise on shock arrival indicates the establishment of the shock wave into the target. The rapid rise in the front gauge indicates a good gauge alignment followed by a levelling of the shock front in the form of a plateau, the Hugoniot stress. An overshoot in longitudinal stress was observed after the initial rise with some ringing attributed to electrical effect.

The voltages were converted into stresses following a technique developed by Rosenberg et al. [22]. The front gauge measured the stress in the PLA while the rear gauge recorded that in the PMMA (backing material). To characterise a material mechanical shock response, five key parameters are required and these are: shock velocity $U_{s}$, particle

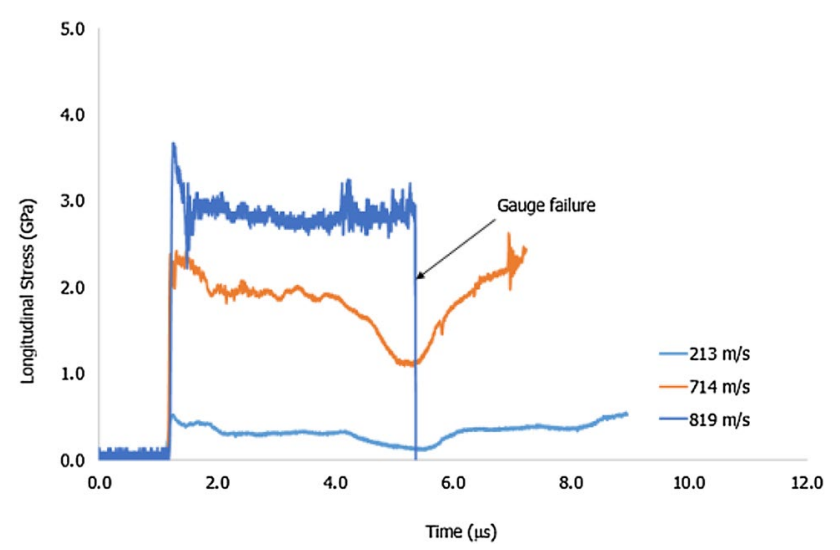

Fig. 5 Representative wave traces of printed PLA shots at 213, 714 and $819 \mathrm{~m} / \mathrm{s}$ (corresponding to impact pressures of 0.30, 1.93 and $2.88 \mathrm{GPa}$ respectively) velocity $U_{p}$, density $\rho$, pressure $\mathrm{P}$, and internal energy $\mathrm{E}$. By employing the principles of conservation of energy, mass and momentum, other parameters can also be obtained from just two parameters. In this study, the $U_{s}$ and $U_{p}$ values were obtained and employed to derive a shock Hugoniot equation of state for the printed PLA. The shock velocity was obtained by calculating the time taken for the shock to travel between the front and rear gauge indicated by the rise in both gauges divided by the known gauge separation distance, with particle velocity determined via the impedance matching technique (requiring knowledge of impactor properties and velocity [23] and subsequently converted to volume asrequired. In addition, the embedded gauges provided a direct measure of in-material stress. A Typical trace for the front and rear gauges of a printed $10 \mathrm{~mm}$ PLA with impact velocity of $714 \mathrm{~m} / \mathrm{s}$ is presented in Fig. 6 .

By employing well-defined flyer materials with an established pressure-particle velocity $\left(P-U_{p}\right)$, and known impact velocity, the $U_{p}$ is obtained graphically via impedance matching technique described extensively by Meyers in Ref. [23]. The $U_{s}-U_{p}$ Hugoniot relationship for the printed PLA was measured/calculated with data points obtained from shock states derived via experimental plate impact experiment. A summary of the experimental conditions and key results where $U_{s}-U_{p}$ data were extracted are presented in Table 3.

Although the samples were observed to contain some degree of porosity, this is quite minimal $(<8 \%)$. As such, the Mie-Gruneisen EOS relationship for porous materials, described in Ref. [23] which predicts volume increases at all pressure for a porosity of $50 \%$ cannot be employed here. Essentially, when the main bulk of the material contains a significant fraction of voids, such as solid foam (pg. 64 of Ref. [24]) with larger variations in impedance, the nature of the response is different. However, for the samples printed here, the void observed is in the quite small, much lower

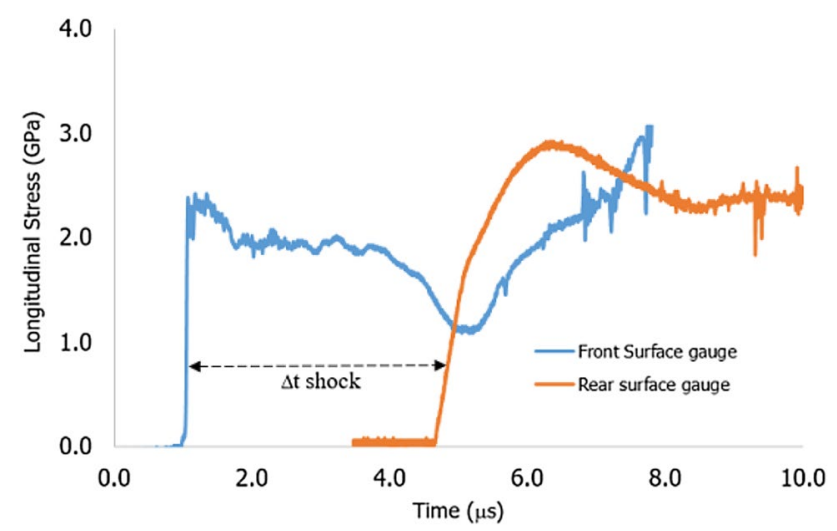

Fig. 6 Typical gauge trace for $10 \mathrm{~mm}$ thick copper impacting a 10.12$\mathrm{mm}$ thick printed PLA at $567 \mathrm{~m} / \mathrm{s}$ 
Table 3 Summary of plate impact experimental result

\begin{tabular}{lllllllll}
\hline $\begin{array}{l}\text { Impact veloc- } \\
\text { ity }(\mathrm{m} / \mathrm{s})\end{array}$ & $\begin{array}{l}\text { Flyer thickness } \\
(\mathrm{mm}) / \text { material }\end{array}$ & $U_{p}(\mathrm{~mm} / \mu \mathrm{s})$ & $U_{s}(\mathrm{~mm} / \mu \mathrm{s})$ & Error $(+)$ & Error $(-)$ & $\mathrm{P}(\mathrm{GPa})$ & Error $(+)$ & Error $(-)$ \\
\hline 213 & $10 / \mathrm{Al}$ & 0.18 & 1.87 & 0.09 & 0.05 & 0.30 & 0.02 \\
312 & $10 / \mathrm{Cu}$ & 0.30 & 2.04 & 0.15 & 0.13 & 0.70 & 0.1 & 0.02 \\
490 & $10 / \mathrm{Al}$ & 0.41 & 2.37 & 0.14 & 0.12 & 1.45 & 0.05 \\
714 & $10 / \mathrm{Al}$ & 0.58 & 2.74 & 0.24 & 0.21 & 1.93 & 0.14 \\
819 & $10 / \mathrm{Cu}$ & 0.73 & 2.93 & 0.21 & 0.11 & 2.88 & 0.11 & 0.12 \\
925 & $10 / \mathrm{Cu}$ & 0.84 & 3.17 & 0.22 & 0.14 & 3.59 & 0.13 & 0.13 \\
\hline
\end{tabular}

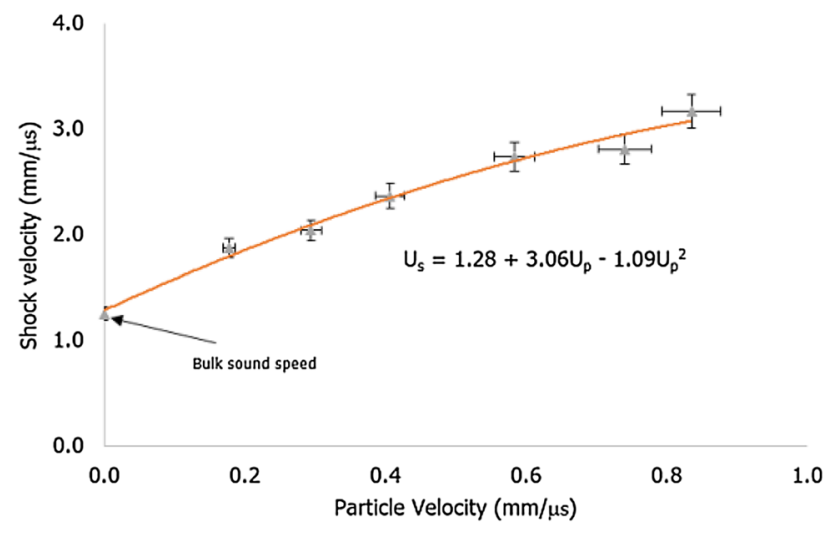

Fig. 7 Non-linear Hugoniot response for printed PLA at low shock velocity ranges

Table 4 Equations of State considered for PLA Hugoniot

\begin{tabular}{lll}
\hline Type & Equation & $\mathrm{R}^{2}$ value \\
\hline Linear form & $U_{S}=1.4+2.02 U_{p}$ & 0.983 \\
Non-linear form & $U_{S}=1.28+3.06 U_{p}-1.09 U_{p}^{2}$ & 0.995 \\
\hline
\end{tabular}

than the width of the pressure gauges employed. Hence, the authors consider that the effect of the voids on the resultant EOS is negligible. Moving forward, both linear and non-linear polynomial fits to the experimental data were considered. For the linear relationship, best-fit to the experimental data, $C_{o}$ and $S$ were found to equal 1.38 and 2.0 respectively, with a residual " $\mathrm{R}$ " " value of 0.983 . However, a 2 nd order polynomial fit produced a higher $\mathrm{R}^{2}=0.995$ which justified its use in Fig. 7. In this case, the polynomial coefficient for the best fit were: $C_{o}=1.28 \mathrm{~mm} / \mathrm{us}, \mathrm{S}=3.06$ and $\mathrm{Q}=-1.09 \mathrm{~mm} / \mathrm{us}$ something highlighted in Table 4. Such non-linear response has been reported in the low pressure (below the 20-30 GPa transition) regime for polymeric materials such as PMMA [25], polyethylene and PVC [26]. However, above the particle velocity of ca. $0.2 \mathrm{~mm} / \mu \mathrm{s}$, as recorded in this experiment, most polymeric materials give a linear response, of the form $U_{s}=C_{o}+S U_{p}$, along with other commercially available polymers and specially produced plastics [9]. The empirical constants $C_{0}$ and $S$ in the linear and polymeric best-fit equations are related to the bulk sound speed (in metals such as copper) [27] and the rate of change of compressibility of the material with pressure [9], respectively. More striking, and in line with other polymeric materials such as PMMA, and polycarbonate [8], is the failure of the experimental data to extrapolate to the zero-pressure ultrasonic measurement as observed in metals. For the non-linear fit considered, the difference between the ultrasonic sound speed and the experimentally derived $C_{0}$ is only $0.02 \mathrm{~mm} / \mu \mathrm{s}$. As such, the bulk sound speed of the material lies only slightly below the zero vertical intercept of the $U_{s}-U_{p}$ plot giving a polynomial non-linear response of the form $U_{s}=C_{o}+S U_{p}+Q U_{p}^{2}$ [28]. This accounts for the narrow variation in $\mathrm{R}^{2}$ value between the linear and non-linear fits.

Such non-linear response has been attributed elsewhere to the two-dimensional nature of polymer compression and to the form of the inter-chain interaction potential [8] owing to the substantial difference in magnitude between the backbone and inter-chain forces as described earlier. Fundamentally, weaker inter-chain forces are overcome during the initial stages of compression before the backbone covalent bonds, thereby resulting in a two-stage compression. A non-linear response occurs at low particle velocities, $<0.2 \mathrm{~mm} / \mu \mathrm{s}$; whereas a more conventional linear $U_{s}-U_{p}$ response is established above $0.2 \mathrm{~mm} / \mu \mathrm{s}$ as described earlier. Comparison (low range data) of the measured $U_{s}-U_{p}$ plot with some selected polymers such as PE and PVC in Fig. 8 revealed similarities in terms of lower bulk sound speed. Such changes have been linked to changes in the density and modulus due to molecular rearrangement of the chains [27].

As can be seen from Fig. 8, PLA lies at the bottom of the plot followed closely by PE with a simple polymer structure. This response could be attributed to the presence of the methyl group on the carbon backbone of the PLA (in Fig. 1) making the chain bulkier thus, providing a steric impediment to flow. Further, the presence of oxygen atom to the carbon chain increases the strength of the carbon-ligand bond and decreases Van-der-Waals forces between chains. Above the $\mathrm{PE}$ response are the highly electronegative polymers PCTFE 


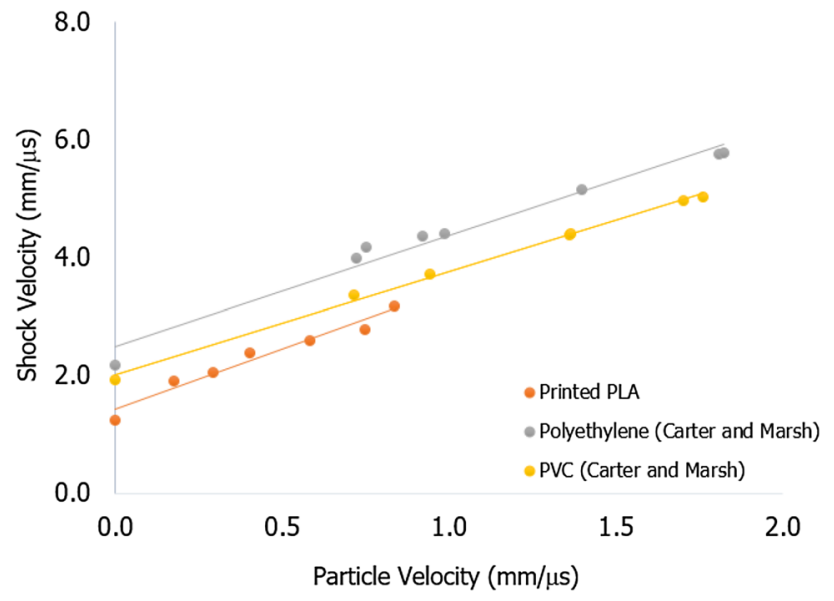

Fig. 8 Comparison of $\mathrm{U}_{\mathrm{s}}-\mathrm{U}_{\mathrm{p}}$ plot of printed PLA with Polyethylene and PVC

and PVC with branch or side chains replaced by electronegative atoms such as fluorine or chlorine. Each of these polymers has other elements introduced onto the carbon backbone or into the side group chains increasing the density throughout the series. The PCTFE, for example, is higher up because of the presence of both chlorine and fluorine which increases the repulsion force as well as the density. As such, the combination of density and strength ensures that the Hugoniot lies above their hydrocarbon neighbours. By employing the non-linear equations of state from Fig. 7 , the hydrodynamic pressure can be calculated according to Eq. 5. When plotted with the measured stresses in Fig. 10, the strength of the material can be predicted.

$P=\rho_{o} U_{p} U_{s}$

From Fig. 9, there appears to be a deviation between pressure as particle velocity increases. This implies that, at least, within the shock pressure regime investigated, the printed

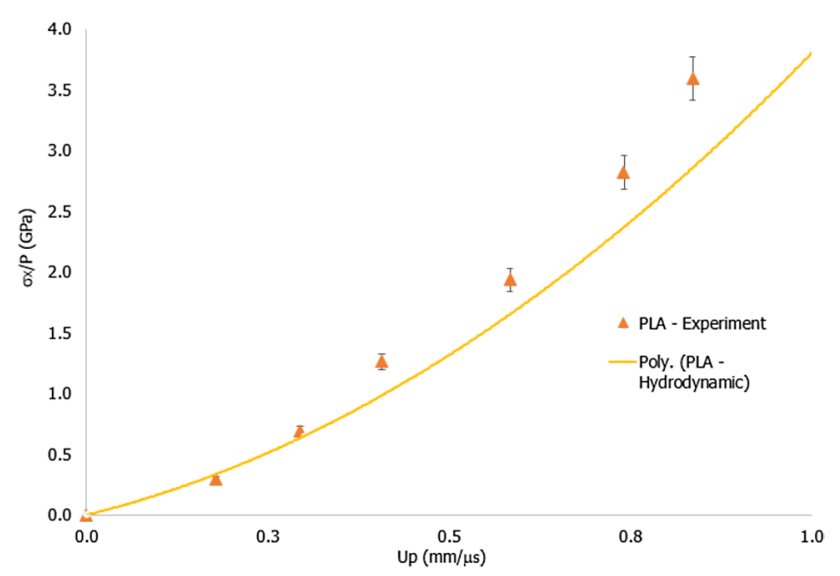

Fig. $9 \sigma_{\mathrm{X} /} \mathrm{P}-\mathrm{U}_{\mathrm{p}}$ Hugoniot relationship for printed PLA plus hydrodynamic response based on Eq. 5

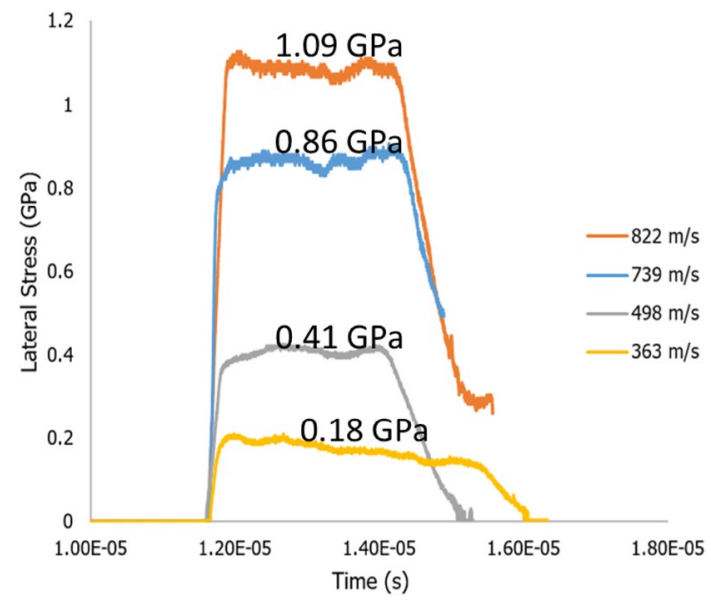

Fig. 10 Lateral stress histories for printed PLA at varying impact velocities; gauges are $4 \mathrm{~mm}$ from the impact surface

PLA appears to strengthen behind the shock front suggesting material strengthening $(\tau)$ according to the relationship in Eq. 6 [29], Similar response where divergence was observed between measured longitudinal stress and calculated hydrodynamic pressures has been recorded with other polymers such as PE, PCTFE and PVC [6] where large deviations were seen. Although, it's interesting to note that Wood et al. [14] observed no deviation between the hydrodynamic and experimental measured parameters for SC-1008 at pressures below $4.00 \mathrm{GPa}$, showing that such a response is not guaranteed-although, above $4 \mathrm{GPa}$, deviations from the hydrostat were observed suggesting potential material strengthening.

$\sigma_{x}=P+\frac{4}{3} \tau$

Recorded lateral gauge traces are presented in Fig. 10 with the corresponding stresses noted on each trace. All traces reveal some similarities; a rapid rise to the Hugoniot stress signifying shock has been induced in the material and a subsequent descent in the trace due to the interaction of the reflected waves with the incident. However, it appears that the response from the different gauge traces shows some marked differences. The recorded gauge traces at 363 and $822 \mathrm{~m} / \mathrm{s}$ reveals that the lateral stress is decreasing. This implies that the material hardens behind the shock front, following Eq. 3. However, we have also observed increasing lateral stresses with the trace labelled $0.86 \mathrm{GPa}$ and undulating stress in the $0.41 \mathrm{GPa}$ trace. The reason for the latter trace is not clear but the authors believe that this is likely a result of the layers of air and material which the shock fronts meet as it travels (as it equilibrates) along the lateral halves of the target in view of the concentric pattern employed in the FDM built parts. This can be verified by performing a similar experiment using the bulk material. However, usefully, the decreasing lateral stresses recorded 
in the 1.09 and $0.18 \mathrm{GPa}$ traces, reveals that the material is hardening behind the shock front, consistent with other polymeric material, such as PMMA [7] and Polycarbonate [30]. This has been attributed to an increase in the proximity of molecular components, arising from the densification of molecules, with strong attractive bonding and steric [31] entangling which tends to resist further strain with time [24].

Another interesting feature is the gauge response time. It was observed that the time taken for the gauge to respond differs with impact velocity. It was expected that the higher impact velocities will produce lower response time but this is not completely seen here. The highest response time of $2.95 \mathrm{E}-7 \mathrm{~s}$ was recorded for the highest impact velocity of $822 \mathrm{~m} / \mathrm{s}$ followed closely by $2.56 \mathrm{E}-7 \mathrm{~s}$ recorded at $498 \mathrm{~m} / \mathrm{s}$ and $1.008 \mathrm{E}-7 \mathrm{~s}$ response time for the $739 \mathrm{~m} / \mathrm{s}$ impact velocity shot. Although differences between response time are insignificant, the authors believe the variations are due to possibly misalignment of the gauges in the lateral half of the target. A slight misalignment of the gauge could increase the response time because of the time it takes for the shock to equilibrate across the gauge length. Although the initial rise in the gauge traces has been attributed elsewhere to gauge equilibrium [32], essentially, polymer densify to large strain against weaker inter-chain Van-der-Waals [24] forces before material begin to exhibit a steady state behind the shock front. This implies that the stress state stability can only be achieved once the polymer has been loaded to compression at which the carbon atoms interact at a maximum density which suggests that rate of compression determines the amplitude and time to stable state. At lower stresses such as that observed in the $0.405 \mathrm{GPa}$, the printed PLA takes more time to equilibrate to the stable state. As such, the effect of densification, which also includes closure of the pores and rearrangement of the molecule to a fully dense state is more visible at lower stresses.

The shear strength data calculated from Eq. 3, using lateral stress gauges at $4 \mathrm{~mm}$ from the impact surface is presented in Table 5.

From Table 5, it is apparent that shear strength increases with increasing impact stresses in agreement with similar polymeric materials [7]. The lateral stresses immediately behind the shock front have been used, along with the known

Table 5 Experimental conditions and results of lateral stress gauges at $4 \mathrm{~mm}$ from the impact face

\begin{tabular}{lllll}
\hline $\begin{array}{l}\text { Flyer thickness } \\
(\mathrm{mm}) / \text { material }\end{array}$ & $\begin{array}{l}\text { Impact } \\
\text { velocity } \\
(\mathrm{m} / \mathrm{s})\end{array}$ & $\sigma_{x}(G P a)$ & $\sigma_{y}(G P a)$ & $2 \tau(\mathrm{Gpa})$ \\
\hline $10 / \mathrm{Cu}$ & 363 & 0.90 & 0.18 & 0.72 \\
$10 / \mathrm{Cu}$ & 478 & 1.32 & 0.41 & 0.91 \\
$10 / \mathrm{Cu}$ & 739 & 2.54 & 0.86 & 1.68 \\
$10 / \mathrm{Cu}$ & 822 & 2.99 & 1.09 & 1.90 \\
\hline
\end{tabular}

longitudinal stresses to determine the shear strength of the printed PLA. Usefully, the shear strength can be employed to help define the elastic-plastic transition. Above the elastic limit, the material behaves plastically and vice versa. By comparing the yield strength of the material to an elastic prediction given in Eq. 7, the Hugoniot Elastic Limit (HEL) can be obtained. This technique has been previously validated in Ref. [7] and employed to determine the HEL of PMMA. Employment of the technique in the determination of the shear strength is presented in Fig. 11. The elastic behaviour is represented by the solid line according to Eq. 7 [24].

$2 \tau=\frac{1-2 v}{1-v} \sigma_{x}$

where " $v$ " is the poisons ratio. A linear line of best-fit has been put through the measured stress which intercepts the elastic prediction at longitudinal stress of $\sim 0.9 \mathrm{Gpa}$. This can be taken as the Hugoniot Elastic Limit. In comparison with similar polymeric material like PMMA where the elastic limit has been placed in the range $\sim 0.7-0.9 \mathrm{GPa}$. It is pertinent to note that the specimens employed here were fabricated with the build direction perpendicular to the shock direction and considering the fact that the mechanical properties of FDM built parts varies with built parameters [33] as well as the bulk material, the elastic limit obtained may vary with the different print settings and the bulk material; although this is only speculative.

From Fig. 11, it is apparent that shear strength increases with stress, as with other similar polymeric materials, PE and PVC. It is however interesting to note that the measured HEL of PLA is within similar range with PMMA which has been shown to have higher strength than basic polymers (particularly PE and PVC). This could be attributed in some ways to steric effect, arising from large chain structure due to the presence of multiple side chains and the presence of electronegative atom in the side group chain. While the presence of oxygen increases the electronegativity, allowing polar attraction to increase the bond strength, it's very likely that the dynamic response is controlled majorly by

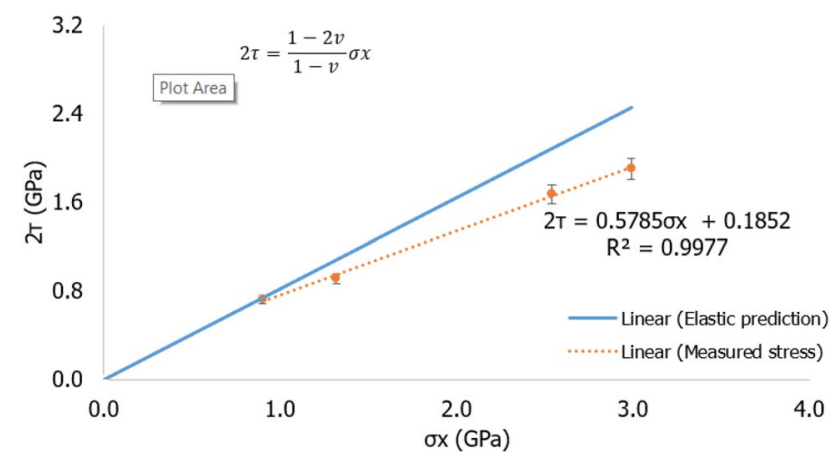

Fig. 11 The Shear strength versus longitudinal stress for Printed PLA 
the interaction between adjacent polymer chains emanating from the inclusion of Methyl group and oxygen to the polymer chain, causing further tangling of the polymer chain structure.

\section{Conclusion}

Using the plate impact experiments, the dynamic response of 3D printed polylactic acid has been investigated over the impact velocity range of $200-900 \mathrm{~m} / \mathrm{s}$ (corresponding to impact stresses of 0.30 to $4.00 \mathrm{GPa}$, respectively). A total of six plate-impact experiments were undertaken to determine the Hugoniot of commercially produced 3D printed PLA via the filament deposition technique. Both linear and non-linear Hugoniot was considered with the non-linear $\mathrm{R}^{2}$ value tending to 1 , giving it a non-linear equation of the form $U_{s}=1.28+3.05 U_{p}-1.09 U_{p}^{2}$. This non-linearity appears indicative of the underlying material structure. A change in slope in the plot of shear strength $(2 \tau)$ at $0.7 \mathrm{GPa}$ against longitudinal stress at $0.90 \pm 0.05 \mathrm{GPa}$ within similar range with PMMA was observed, attributed to both the increase chain entanglement and electronegativity of the polymer chain structure via the addition of methyl and oxygen atom respectively to the polymer chain.

Acknowledgements The authors wish to thank Mr Andrew Roberts and Mr David Miller for their assistance in undertaking the experimental work. This work was conducted as part of the PhD studies of the primary author, Henry Agu who was sponsored by Nigerian Air Force Grant under the leadership of Air Marshal Sadiq Abubakar, the Chief of the Air Staff.

Author Contributions HA designed, coordinated this research and drafted the manuscript, GJ Appleby-thomas conceived and carried out data analysis, AH participated in the research coordination and DW assisted with the analysis of the data obtained. The authors read and approved the final manuscript.

Funding This research was funded by the Chief of the Air Staff, Nigerian Air Force under the Grant Number 2016/2901/PHD.

Availability of Data and Materials All datasets and software used for supporting the conclusion of this article are available on demand.

\section{Compliance with Ethical Standards}

Conflict of interest The authors declare they have no competing interests.

Open Access This article is distributed under the terms of the Creative Commons Attribution 4.0 International License (http://creativeco mmons.org/licenses/by/4.0/), which permits unrestricted use, distribution, and reproduction in any medium, provided you give appropriate credit to the original author(s) and the source, provide a link to the Creative Commons license, and indicate if changes were made.

\section{References}

1. Lasprilla AJR, Martinez GAR, Lunelli BH, Jardini AL, Filho RM (2012) Poly-lactic acid synthesis for application in biomedical devices-A review. Biotechnol Adv 30(1):321-328

2. Field JE, Walley SM, Proud WG, Goldrein HT, Siviour CR (2004) Review of experimental techniques for high rate deformation and shock studies. Int J Impact Eng 30(7):725-775

3. Giordano RA, Wu BM, Borland SW, Cima LG, Sachs EM, Cima MJ (1997) Mechanical properties of dense polylactic acid structures fabricated by three dimensional printing. J Biomater Sci Polym Ed 8(1):63-75

4. Song Y, Li Y, Song W, Yee K, Lee KY, Tagarielli VL (2017) Measurements of the mechanical response of unidirectional 3D-printed PLA. Mater Des 123:154-164

5. Carneiro OS, Silva AF, Gomes R (2015) Fused deposition modeling with polypropylene. Mater Des 83:768-776

6. Millett JCF, Brown EN, Gray GT, Bourne NK, Wood DC, Appleby-Thomas G (2016) The effects of changing chemistry on the shock response of basic polymers. J Dyn Behav Mater 2(3):326-336

7. Millett JCF, Bourne NK (2000) The deviatoric response of polymethylmethacrylate to one-dimensional shock loading. J Appl Phys 88(12):7037-7040

8. Carter W, Marsh P Hugoniot equationof state of polymers

9. Bourne NK (2016) On the shock response of polymers to extreme loading. J Dyn Behav Mater 2(1):33-42

10. Kim E, Shin Y-J, Ahn S-H (2016) The effects of moisture and temperature on the mechanical properties of additive manufacturing components: fused deposition modeling. Rapid Prototyp J 22(6):887-894

11. Sood AK, Ohdar RK, Mahapatra SS (2010) Parametric appraisal of mechanical property of fused deposition modelling processed parts. Mater Des 31(1):287-295

12. Gebisa AW, Lemu HG (2019) Effect of process parameters on compressive properties of ULTEM 9085 Produced by FDM Process, p. V002T02A056

13. Popovich A, Sufiiarov V, Polozov I, Borisov E, Masaylo D, Orlov A (2016) Microstructure and mechanical properties of additive manufactured copper alloy. Mater Lett 179:38-41

14. Wood DC, Hazell PJ, Appleby-Thomas GJ, Barnes NR (2011) Shock behaviour of a phenolic resin. J Mater Sci 46(18):5991-5999

15. Hazell PJ, Appleby-Thomas GJ, Trinquant X, Chapman DJ (2011) In-fiber shock propagation in Dyneema ${ }^{\circledR}$. J Appl Phys 110(4):043504

16. Appleby-Thomas GJ, Hazell PJ, Stennett C, Cooper G, Helaar K, Diederen AM (2009) Shock propagation in a cemented tungsten carbide. J Appl Phys 105(6):064916

17. Appleby-Thomas GJ, Hazell PJ, Sheldon RP, Stennett C, Hameed A, Wilgeroth JM (2014) The high strain-rate behaviour of selected tissue analogues. J Mech Behav Biomed Mater 33(1):124-135

18. Millett JCF, Lowe MR, Appleby-Thomas G, Roberts A (2016) The mechanical and optical response of polychlorotrifluoroethylene to one-dimensional shock loading. Metall Mater Trans A 47(2):697-705

19. Ahn SH, Montero M, Odell D, Roundy S, Wright PK (2002) Anisotropic material properties of fused deposition modeling ABS. Rapid Prototyp J 8(4):248-257

20. Parker NG, Mather ML, Morgan SP, Povey MJW (2010) Longitudinal acoustic properties of poly(lactic acid) and poly(lactic-coglycolic acid). Biomed Mater 5(5):055004

21. Wood A (1930) A textbook of sound: being an account of the physics of vibrations with special reference to recent theoretical 
and technical developments by Wood, A. B. (Albert Beaumont). Macmillan, New York

22. Rosenberg Z, Yaziv D, Partom Y (1980) Calibration of foil-like manganin gauges in planar shock wave experiments. J Appl Phys $51: 3702$

23. Meyers MA (2014) Dynamic behavior of material. Wiley, Hoboken

24. Bourne N (2013) Materials in mechanical extremes; fundamentals and application. Cambridge University Press, Cambridge

25. Barker M, Hollenbach LE (1970) Shock-wave studies of PMMA, fused silica, and sapphire. J Appl Phys 41:4208-4226

26. Dattelbaum DM, Coe JD (2016) The dynamic loading response of carbon-fiber-filled polymer composites. In: Silberschmidt VV (ed) Dynamic deformation, damage and fracture in composite materials and structures. Woodhead Publishing, Sawston

27. Davison L, Graham RA, Davison L, Graham RA (1979) Shock compression of solids. Phys Rep 55(4):255-379

28. Hazell PJ (2016) Shock loading of polymer composites. Elsevier, Amsterdam
29. Rosenberg Z, Bourne NK, Millett JCF (2007) On the effect of manganin gauge geometries upon their response to lateral stress. Meas Sci Technol 18(7):1843-1847

30. Millett JCF, Bourne NK (2006) Shock and release of polycarbonate under one-dimensional strain. J Mater Sci 41(6):1683-1690

31. Shepherd CJ, Appleby-Thomas GJ, Wilgeroth JM, Hazell PJ, Allsop DF (2011) On the response of ballistic soap to one-dimensional shock loading. Int J Impact Eng 38(12):981-988

32. Painter JD et al (2012) On the importance of encapsulation environment for lateral gauges. AIP Conf Proc 1426:454-457

33. Alharbi N, Osman R, Wismeijer D (2016) Effects of build direction on the mechanical properties of 3D-printed complete coverage interim dental restorations. J Prosthet Dent 115(6):760-767

Publisher's Note Springer Nature remains neutral with regard to jurisdictional claims in published maps and institutional affiliations. 